\title{
Micrometer-thickness liquid sheet jets flowing in vacuum
}

\author{
Gediminas Galinis, ${ }^{1, a)}$ Jergus Strucka, ${ }^{1}$ Jonathan C. T. Barnard, ${ }^{1}$ Avi Braun, ${ }^{2}$ Roland A. Smith, ${ }^{3}$ and \\ Jon P. Marangos ${ }^{1}$ \\ 1) Quantum Optics and Laser Science Group, Imperial College London, London, SW7 $2 A Z$, \\ United Kingdom. \\ ${ }^{2)}$ The Experimental Solid State Physics Group, Imperial College London, London, SW7 $2 A Z$, \\ United Kingdom. \\ 3) Plasma Physics Group, Imperial College London, London, SW7 2AZ, United Kingdom.
}

(Dated: 31 July 2017)

Thin liquid sheet jet flows in vacuum provide a new platform for performing experiments in the liquid phase, for example X-ray spectroscopy. Micrometer thickness, high stability and optical flatness are the key characteristics required for successful exploitation of these targets. A novel strategy for generating sheet jets in vacuum is presented in this article. Precision nozzles were designed and fabricated using high resolution $(0.2 \mu \mathrm{m})$ 2-photon 3D printing and generated $1.49 \pm 0.04 \mu \mathrm{m}$ thickness, stable and $<\lambda / 20$-flat jets in isopropanol under normal atmosphere and under vacuum at $5 \times 10^{-1}$ mbar. The thin sheet technology also holds great promise for advancing the fields of high harmonic generation in liquids, laser acceleration of ions as well as other fields requiring precision and high repetition rate targets.

PACS numbers: $47.60 . \mathrm{Kz}, 47.15 . \mathrm{Uv}$

\section{INTRODUCTION}

The liquid phase is a fundamental state of matter facilitating a vast range of chemical and biochemical reactions. To date the electronic structure of liquids has been studied by means of photoelectron spectroscopy ${ }^{1-4}$ (PES), Xray absorption spectroscopy ${ }^{5-7}$ (XAS), resonant inelastic X-ray scattering 8 (RIXS) as well as transient absorption spectroscopy (TAS) ${ }^{9-11}$. X-ray absorption techniques provide information on the local electronic structure and are sensitive to the chemical environment. However, the methodology is somewhat limited in the soft X-ray (few $100 \mathrm{eV}$ photon) range due to high absorption crosssections at these energies. Micrometer-thickness liquid sheet jets have scope to overcome the limits placed to absorption spectroscopy by high absorption in the soft $\mathrm{X}$-ray range. Further they will greatly improve temporal resolution in transient $\mathrm{X}$-ray absorption measurements by effectively eliminating temporal walk-off between the pump and probe pulses due to dispersion. They also satisfy the requirement that the liquid target should flow rapidly so as to avoid photo-bleaching effects that can result from exposure of molecules to many pulses of either pump or probe. The liquid targets must also be able to operate in vacuum $\left(<10^{-1}\right.$ mbar $)$ to minimise the background absorption of soft X-rays and allow for measurement techniques based on electron and ion emission. A small-scale (single room) laboratory experiment could be created in which an advanced compact soft Xray source $\mathrm{e}^{12}$ is used with a flowing micrometer-thickness liquid jet target to probe ultrafast processes. This approach could complement large scale synchrotron exper-

\footnotetext{
a)g.galinis@imperial.ac.uk
}

iments and could make soft X-ray experiments with liquids accessible to a wider scientific community.

Introduction of a liquid into vacuum is a difficult undertaking, in particular when high vapour pressure liquids are considered. Several schemes have been proposed to address this and have been used with varying degrees of success. For photoelectron spectroscopy circular microjets with diameters typically in the range of 10-25 um have often been used ${ }^{1,2,13}$. For these measurements the smooth and laminar flow region of the jets immediately after the nozzle are used before the liquid stream breaks up into droplets due to Rayleigh instabilities. An alternative approach is to use liquid flow cells $(0.5-2 \mu \mathrm{m})$, where the liquid is encapsulated/flows between 100 nm-thickness $\mathrm{SiN}$ or $\mathrm{SiC}$ windows - an approach that has typically been implemented for X-ray absorption techniques ${ }^{6,7,11}$. In addition to circular and cell-targets, free flowing flat liquid jets (100 $\mathrm{mm}$ thickness) can be used for X-ray absorption measurements ${ }^{9}$. Compared to liquid cells these targets have the key advantage of eliminating absorption in the windows. At atmosphere several examples of thin liquid targets have been reported ${ }^{14,15}$. These jets have been enabled by manufacturing specialised nozzles to generate liquid sheets. An example of a micrometer-scale liquid jets operating in vacuum has been reported recently ${ }^{16}$. The technological concept comprised two circular microjets aligned to cross at an angle ${ }^{17}$ in vacuum and a $1.2 \mu \mathrm{m}$-thick sheet jet of water was generated in the collision region.

The use of micrometer-thickness liquid sheet jets is not limited to X-ray absorption. In principle, the technology could be used for angularly resolved photoelectron spectroscopy provided the surface of the jet is sufficiently flat. Likewise, the technology holds great promise for applications of optically flat liquid surfaces as targets for the laser acceleration of ions ${ }^{18}$, and as replaceable or high 
repetition rate plasma mirrors for high power laser contrast enhancement. The self-healing properties of liquids can also be advantageous for processes where high intensity laser damage is unavoidable. Moreover, thin liquid jet technology can potentially be used for high harmonic generation (HHG) in liquids.

Currently, the only working technology for generating few-micrometer-thick liquid sheets in vacuum is the colliding microjet jet system ${ }^{16}$. This technology is expensive and difficult to use in practice due to the requirement to maintain precise alignment of two jets in vacuum at a selected crossing angle. Here we demonstrate an alternative technology to generate optically flat, few-micrometer thickness liquid sheet jets in vacuum through the use of custom 3D-printed nozzles. The single nozzle concept eliminates the colliding jet alignment problem. This technology benefits from direct two-photon laser writing manufacturing: high resolution $(0.2 \mu \mathrm{m}), 3 \mathrm{D}$ printed nozzles were designed, manufactured and tested with isopropanol as a working fluid. Interferometric flatness and thickness characterisation was also undertaken.

\section{METHODS}

\section{A. 3D printing of nozzles}

The purpose of this work was to create nozzle geometries that would generate an optically flat and very thin $(<5 \mu \mathrm{m})$ liquid sheet jet for use in attosecond timescale pump-probe experiments with soft X-ray photons of energy in the range 150 to $600 \mathrm{eV}$. The single nozzle design eliminates many of the problems inherent in colliding circular jet systems used to create thin liquid sheet jets in vacuum ${ }^{16}$. Custom made nozzles were designed using CAD software and 3D-printed using a high resolution $(0.2 \mu \mathrm{m})$ two-photon absorption printer (Nanoscribe $\mathrm{GmbH}$ ) with an IP-S polymer material. Typically a drop of IP-S material was deposited onto a metal substrate, that was loaded into the printer. Then the microscope objective of the Nanoscribe was immersed in the printing fluid and the printing process commenced. A localised volume of printing fluid was exposed to laser light causing polymerisation and a nozzle geometry was written as the objective scanned through the fluid. Due to the high resolution requirement and to minimise the total printing time an aluminium nozzle substrate was pre-machined mechanically and only the final slit assembly (the nozzle) was 3D printed onto this. The outter dimensions of the nozzle body were $1000 \times 800 \times 700 \mu \mathrm{m}$ and the nozzle was printed block by block such that each block was of a size of $300 \times 300 \times 250 \mu \mathrm{m}$. Further time was saved by printing only the shell and scaffolding of the geometry and not exposing every point of the geometry to laser light. Thus unexposed printing fluid was trapped within the printed shell. After printing the nozzles were washed with PGMEA for 20 min and subsequently rinsed with isopropanol for another $2 \mathrm{~min}$. The nozzle plate to-

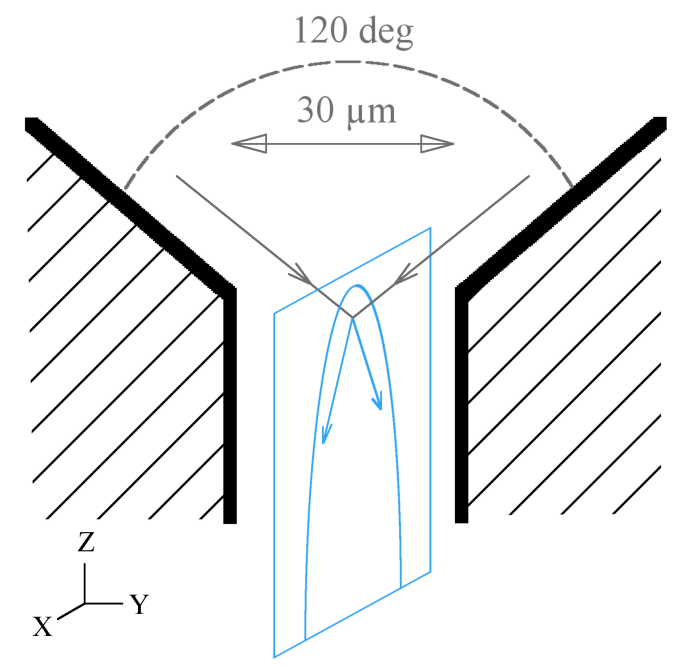

FIG. 1. Schematic drawing of the nozzle cross-section (ZYplane). The nozzle geometry guides liquid stream lines to collide within the nozzle at a $120^{\circ}$ angle and upon collision a flat sheet jet is formed (within ZX-plane) when liquid exits the nozzle ${ }^{17}$. The sheet jet plane is perpendicular to the crosssection plane.

gether with the printed features were then baked in an oven for 45 min gradually ramping the temperature to $195{ }^{\circ} \mathrm{C}$. This process was required to cure the trapped liquid within the shell and scaffolding. Various nozzle geometries were tested with a nozzle shape similar to that used by Kondoh et. al. ${ }^{15,17}$ where the stream lines of the liquid are guided to collide within the nozzle at a selected angle. Due to momentum conservation a flat liquid jet is then generated upon exiting the nozzle (see Fig.1) .

\section{B. Flow system and pulse damping}

Two versions of the flow system were implemented: i) a hydrostatic pressure driven flow system typically used for normal atmospheric measurements (but in principle not limited to the atmospheric experiments) and ii) an HPLC-pump-driven cyclic flow system typically used for testing nozzles in vacuum and better suited to long run times and recirculation of high-value samples. Only the latter is described in detail in this article as jet performance metrics are very similar for both systems.

Initial tests of liquid flow for various nozzle shape geometries were conducted in normal atmosphere with a hydrostatic pressure driven set-up, where the flow of liquid was induced via compressed He gas. Typically a backing pressure of up to 5 bar was used and a stable flow was established. The flow rate for the compressed-gas-driven jets were in the range of $1-10 \mathrm{~mL} / \mathrm{min}$. Continuous operation up to 20 minutes was achieved (only limited by the size of the liquid reservoir, $200 \mathrm{~mL}$ ). This was sufficient to record images of interference fringes using illumination 
by a $633 \mathrm{~nm}$ He-Ne laser as well as to record white light spectral interference fringes from which absolute thickness can be determined.

After the tests in atmosphere the nozzles were used in the cyclic flow system under vacuum to confirm that stable operation was possible at lower pressure and the jet characteristics remained the same (see Fig. 2). The

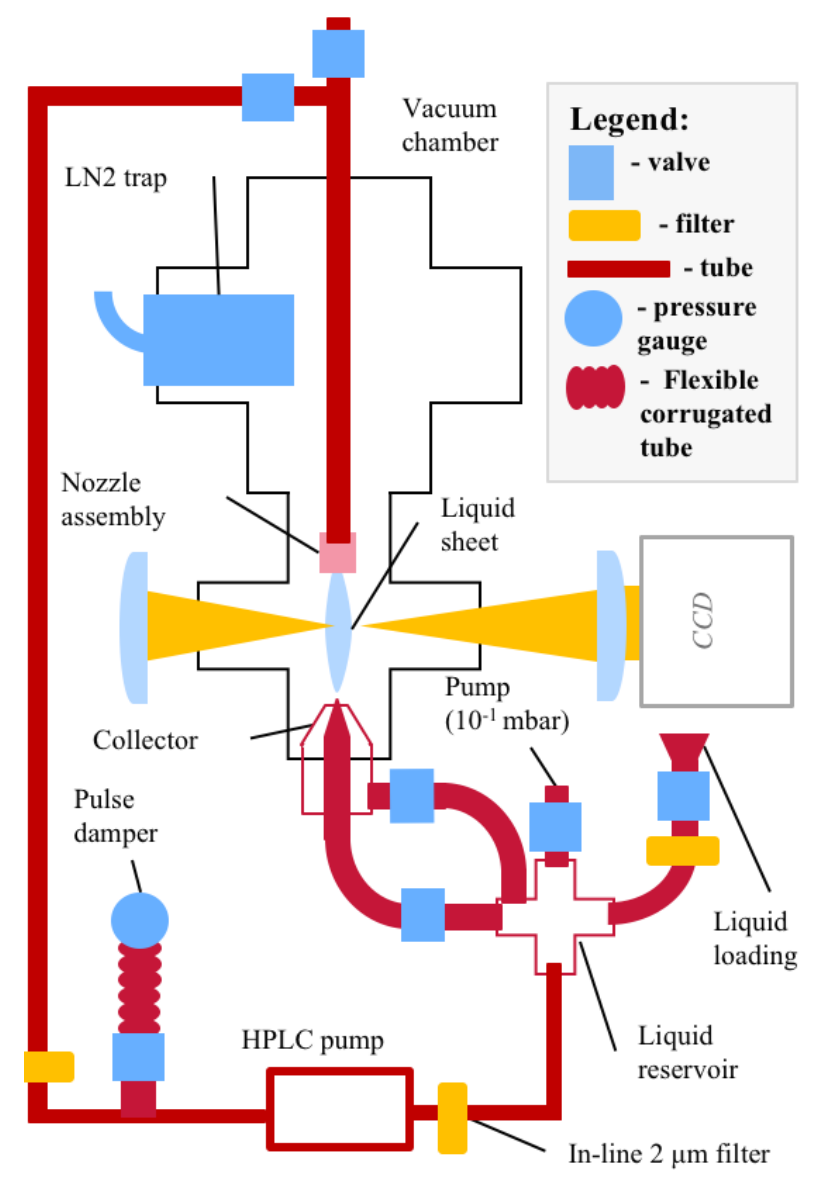

FIG. 2. A schematic of a test vacuum set-up. The system contains a vacuum chamber equipped with $300 \mathrm{~L} / \mathrm{s}$ turbomolecular, $80 \mathrm{~m}^{3} / \mathrm{h}$ forevacuum screw pump and cryogenic liquid nitrogen cooled trap, liquid reservoir, HPLC pump, inline filters $(2 \mu \mathrm{m})$, nozzle assembly, collector and a by-pass arm for collecting liquid during the alignment of the jet. A pressure of $5 \times 10^{-1}$ mbar was achieved with the liquid jet flowing in vacuum.

flow system comprised of a reservoir, flexible tubing, HPLC liquid chromatography pump (Knauer, Azura $6.1 \mathrm{~L})$, pulse damping system, liquid injection and collection assemblies adapted for operation in the vacuum chamber. The reservoir stored a $200 \mathrm{~mL}$ volume of solvent and it was connected via a flexible tube to the input of the HPLC pump. Solvent in the reservoir was evacuated and out-gassed in situ prior to the operation of the jets in vacuum. The pulse damping system was formed by a length of flexible corrugated tube and a pressure gauge ${ }^{19}$. Unwanted pulsation seen as a variation of the jet length due to the HPLC piston motion was reduced to less than $0.5 \%$ using this simple solution. Pulsation-free flow of liquid was coupled to a 3D-printed nozzle housed in the nozzle holder. The flow of liquid through the nozzle (slit dimensions of $260 \times 30 \mu \mathrm{m}$ ) generated flat sheet jets in vacuum. The liquid was then recycled back to the reservoir. The flow rate of liquid was in the range of $5-12 \mathrm{~mL} / \mathrm{min}$. The generated jet was captured with a conically-shaped double-walled collector, the diameter and length of the collector aperture (diameter of $500 \mu \mathrm{m}$ ) were tuned so that the back streaming of gas would not affect the flow of the jet into the collector ${ }^{20}$. The sheets jets were tested in vacuum using a turbo molecular pump with a pumping speed of $300 \mathrm{~L} / \mathrm{s}$ as well as a cryogenic liquid nitrogen cooled trap with an estimated pumping speed of $\sim 5000 \mathrm{~L} / \mathrm{s}$ to maintain reasonable vacuum levels during the operation of the jet at low pressure. A base pressure of $5 \times 10^{-1}$ mbar was achieved but was limited by the small chamber aperture and resulting small pumping capacity. All results presented in this article are for isopropanol, though the system is capable of operating with a broad range of fluids in principle. Further experimental work and numerical simulations are ongoing to optimise our nozzle designs for good performance with liquids with a range of viscosities and surface tensions.

\section{Nozzle optimisation and operation}

The 3D printing process required a definition and optimisation of various printing parameters so that the resulting features represent the model dimensionally and do not display much shrinkage on curing or collapse after printing. One has to adjust the laser focus scan speed, geometry and dimensions of the scaffolding and walls, i.e. the thickness of the walls, the size of scaffolding cells as well as the stitching angle of the blocks. Since the full object is printed block by block the position of the stitching edge had to be carefully selected so that the imperfections of the joining line do not create unwanted features/instabilities on the surface of the flowing liquid jet. Typically such edges were moved as far as possible from the exit slit. The printing time of each nozzle was of the order of 2 hours. A specialised nozzle plate holder was designed to allow for batch printing of up to 8 nozzles at a time. The nozzle were printed and cured in an oven at a temperature of $195{ }^{\circ} \mathrm{C}$. Thereafter the nozzles were inspected using an optical microscope (see Fig. 3).

The flow of liquid through the nozzle was then tested using a simple compressed He gas system. Nozzles were tested up to a pressure of 8 bar and did not show any structural damage. A more problematic issue was that of clogging due to micro particles that were generated inside the tubing due to tightening or loosening the compression fittings. In-line filters were used to avoid excessive clogging and extended nozzle operation time up to several weeks. The clogged nozzles could typically be cleaned by reverse flowing compressed He gas. 


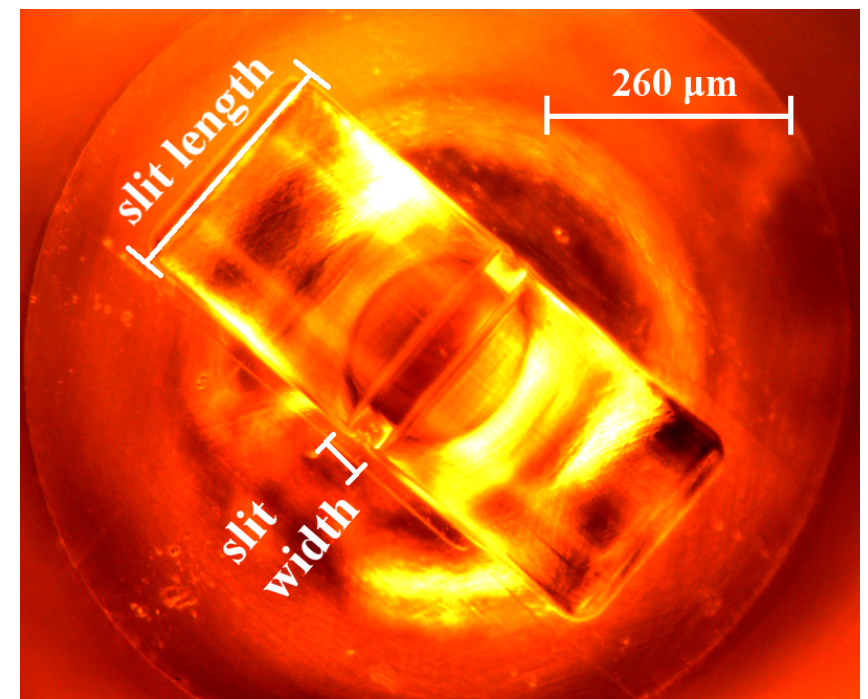

FIG. 3. A microscope image of the printed nozzle. The nozzle slit is aligned along the diagonal from the bottom-left corner to the top-right corner of the image. Here the slit dimensions are $260 \times 30 \mu \mathrm{m}$.

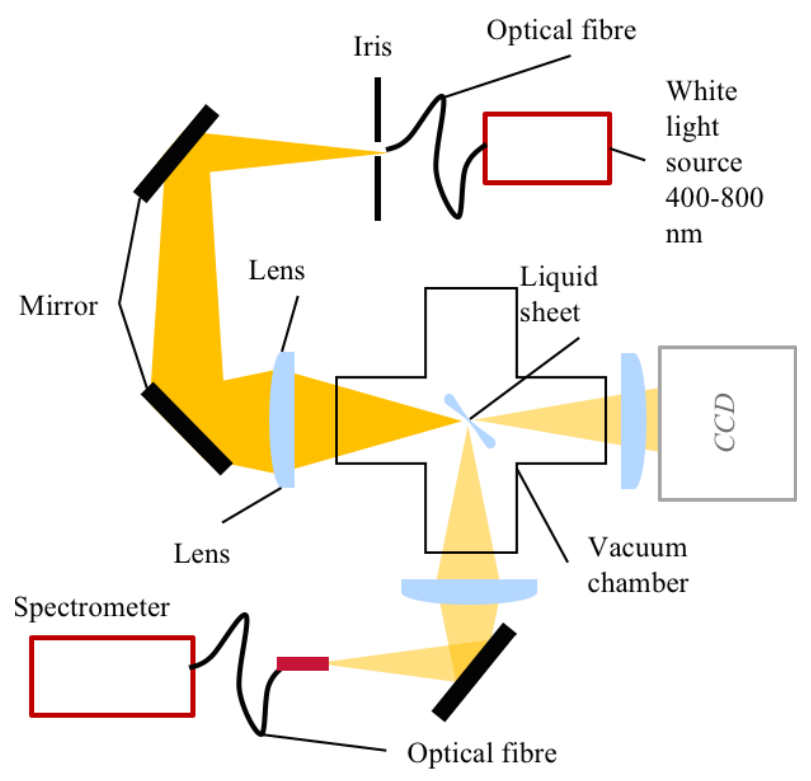

FIG. 4. A schematic of the optical set-up for the white light interferometry. The size of iris aperture defined the spot size of the measurement. In normal atmosphere light was focused with a microscope objective with a much shorter focal distance and a spot size of $75 \mu \mathrm{m}$ was achieved. In the vacuum chamber white light was focused from the outside with a $\mathrm{f}=75 \mathrm{~mm}$ lens and a spot size of about $200 \mu \mathrm{m}$ was measured.

\section{White light and monochromatic interferometry}

The absolute thickness of the jets was measured by white light interferometry (see Fig. 4) while relative thickness and flatness was established from images of in- terference fringes of the jet illuminated with a red $633 \mathrm{~nm}$ He-Ne laser (see Fig. 6).

White light was focused onto a iris to define a spot size of $75 \mu \mathrm{m}$ in air and $200 \mu \mathrm{m}$ in vacuum (due to space constraints for the imaging system) and the light rays from the iris were re-imaged onto the jet at a $45^{\circ}$ half angle of incidence with respect to the surface normal of the liquid stream. The reflected light was focused on an optical fibre that guided the light into a spectrometer (OceanOptics HR4000, 200-1100 nm). The recorded spectral interferograms were analysed to determine jet thickness over an area defined by a spot size of white light on the surface of the jet. A peak finding algorithm was implemented and the positions of the spectral peaks were found by determining a centre-of-mass for each one. The thickness, $d$, of the jet was then calculated from the positions of the adjacent peaks, $\lambda_{1}$ and $\lambda_{2}$, using the following equation:

$$
d=\left(\frac{2\left(n_{1}^{2}-\sin ^{2} \theta\right)^{1 / 2}}{\lambda_{1}}-\frac{2\left(n_{2}^{2}-\sin ^{2} \theta\right)^{1 / 2}}{\lambda_{2}}\right)^{-1}
$$

where $\theta$ is an angle of incidence with respect to the surface normal, $n_{1}$ and $n_{2}$ are the refractive indices at $\lambda_{1}$ and $\lambda_{2}$, respectively. Acquired thickness for each pair of peaks in each spectrum agreed on average within a range of $\pm 5 \%$.

Monochomatic interferometery was undertaken by using a $633 \mathrm{~nm}$ He-Ne laser. Good quality fringe images were achieved when the jet was illuminated at an angle of $15^{\circ}$ with respect to the surface normal. This was the lowest angle at which the laser beam did not clip the microscope objective that was used for imaging. The interferometric images were then analysed to extract the phase and therefore the relative thickness over the selected area of the jet. Monochromatic interferograms were converted into a phase map using a phase retrieval code written by Jason Cole ${ }^{21}$. The resulting phase maps were unwrapped using an algorithm by Goldstein et. al. ${ }^{22}$ and converted into a thickness map.

\section{RESULTS}

\section{A. Stability and flow of the jet in vacuum}

The compressed gas set-up was tested under normal atmosphere and in vacuum. A pulsation free flow was established, however, the set-up could only be used in non-recirculating mode. The flow rates of the jet ranged between $5-10 \mathrm{~mL} / \mathrm{min}$, therefore a reservoir of $200 \mathrm{~mL}$ would empty in 20-40 min. For some applications this duration may be acceptable, however for the transient absorption experiments where multiple laser shots have to be accumulated for each delay stage position up to few hour continuous operation is preferred. This can be achieved either by increasing the reservoir size for the gas driven system or by utilizing a close-loop system. The closed-circulation set-up was implemented and 


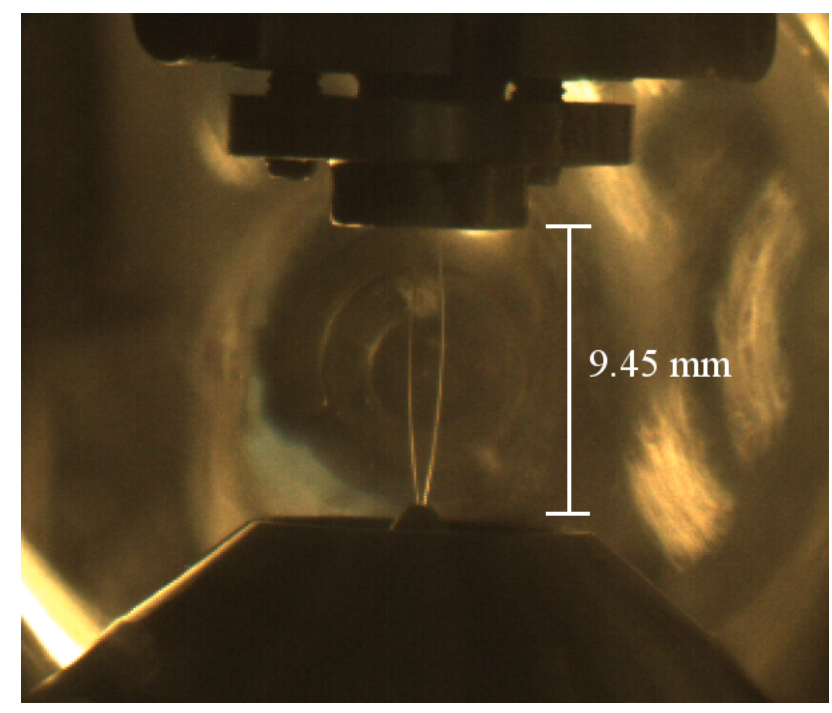

FIG. 5. An image of the liquid isopropanol jet flowing in vacuum. The pressure in the vacuum chamber is $5 \times 10^{-1}$ mbar. The flow rate is $9.4 \mathrm{~mL} / \mathrm{min}$. The jet flows from the nozzle holder assembly (top) to the liquid collector (bottom). The length of the jet is $9.45 \pm 0.05 \mathrm{~mm}$ and the width is $1.61 \pm 0.07 \mathrm{~mm}$. The jet plane is tilted at $45^{\circ}$ with respect to the CCD plane, thus, the jet is $1.14 \pm 0.05 \mathrm{~mm}$ wide in the image. The collector has a secondary tube around it to collect liquid during the alignment of the jet.

tested first. A drawback of the HPLC pump is that it utilizes a piston-driven system which can result in flow rate pulsation. Without a pulsation damping system the length of the jet showed variations of up to $5 \%$ which also meant a corresponding variation of jet thickness an unwanted side effect that is problematic for many applications. With the pulsation damping, the variation was reduced to $<0.5 \%$ in normal atmosphere. The jet flow in vacuum is more difficult to stabilise as the aperture of the liquid collector is effectively open to vacuum pumping and there can be a considerable flow of solvent vapour against the flow of the liquid jet ( $47 \mathrm{mbar}$ vapour pressure at $20^{\circ} \mathrm{C}$ in the reservoir and $5 \times 10^{-1}$ mbar in the vacuum chamber). It was found that a good alignment of the jet with respect to the collector aperture was crucial to establish a stable flow in vacuum (see Fig. 5), the distance of the nozzle to the collector hole had to be tuned within a range of $\pm 150 \mathrm{\mu m}$. The liquid jet was also driven with compressed gas in vacuum and completely pulse-free jet was established. Pressure measurements were undertaken with a pirani gauge located at $10 \mathrm{~cm}$ distance from the jet. A vacuum level of $5 \times 10^{-1} \mathrm{mbar}$ was achieved when the jet was flowing.

\section{B. Optical flatness and surface profile}

The optical flatness of the jet was assessed by analysing the images of the interference fringes of the flowing liquid sheet under normal atmosphere. The interference fringes of the jet were recorded for two different flow rates at 2 bar, $7.4 \mathrm{~mL} / \mathrm{min}$ and ii) at $3 \mathrm{bar}, 9.1 \mathrm{~mL} / \mathrm{min}$ (see a) and b) in Fig. 6, respectively). For the latter the jet was exceptionally flat as can be seen from the nearly parallel fringes along the centre flow line of the liquid jet. The thickness increased sharply at the edges of the sheet, where an approximately circular rim is formed due to the surface tension of the liquid. The fringe spacing uniformly increases going from left to right indicating a gradual decrease of thickness along the full length of the jet (jet dimensions: $5.79 \pm 0.05 \mathrm{~mm}$ long and $0.87 \pm 0.05 \mathrm{~mm}$ wide at the widest part). This is qualitatively consistent with the previous measurements of liquid sheet jets ${ }^{14-16}$. The fringes exhibit slight curvature across the full width of the jet between the two rims (see c) in Fig. 6), which could result in unwanted lensing effect for a larger interaction region. However, in the anticipated applications of the jet the laser beam will be focused to a spot size of $10-100 \mu \mathrm{m}$. Therefore the relevant dimension is then the optical flatness over the range of $100 \mu \mathrm{m}$. Over this length the jet is $<\lambda / 20$ flat at the centre of the flow. The flatness of the jet could be further improved by fine tuning the geometry of the nozzle.

At the higher flow rate the jet dimensions increased: the jet length was $8.93 \pm 0.05 \mathrm{~mm}$ and the jet width was $1.40 \pm 0.05 \mathrm{~mm}$. The overall trend of the flow is similar in character: the thickness decreases along the flow direction and there is a steep well localised increase in thickness around the edges. However, there is another marked difference between the two flow rates - at the higher flow rate the jet shows more pronounced standing waves following a diamond-shaped pattern. The fringe thickness at these locations changes by few percent and proportional changes in liquid thickness are anticipated. The appearance of more pronounced standing waves is potentially attributed to the higher Reynolds number, $R$, at the higher flow rate. Given the physical parameters of isopropanol: density $\rho=786 \mathrm{~kg} / \mathrm{m}^{3}$, dynamic viscosity $\nu=2.37 \mathrm{mPa} \cdot \mathrm{s}$ at $20^{\circ} \mathrm{C}$ as well as the average velocities $(13.6 \mathrm{~m} / \mathrm{s}$ and $16.7 \mathrm{~m} / \mathrm{s})$ and the characteristic slit length dimension of $260 \mu \mathrm{m}$, the esimated Reynolds numbers were 1170 and 1440 for the lower and higher flow rate, respectively. Both values are below the critical threshold of $R<2000$ at which the flow is regarded as turbulent. The transition region is typically in the range of $1800<R<2000$. The Reynolds number for the higher flow is close to the transition regions and potentially leads to the onset of standing waves. Another possible explanation is that the nozzle shape and geometry caused a sharp change in velocity of the jet at the ends of the slits and therefore the standing waves were observed. A more definitive conclusion could be drawn by undertaking full fluid dynamics simulations ${ }^{23,24}$. Even though the standing waves are present in the jet an optically flat region of the size of the laser spot can be located in multiple places across the full area of the sheet jet.

Water jets were tested with the current nozzle design at similar stagnation pressure. However, the surface of the 

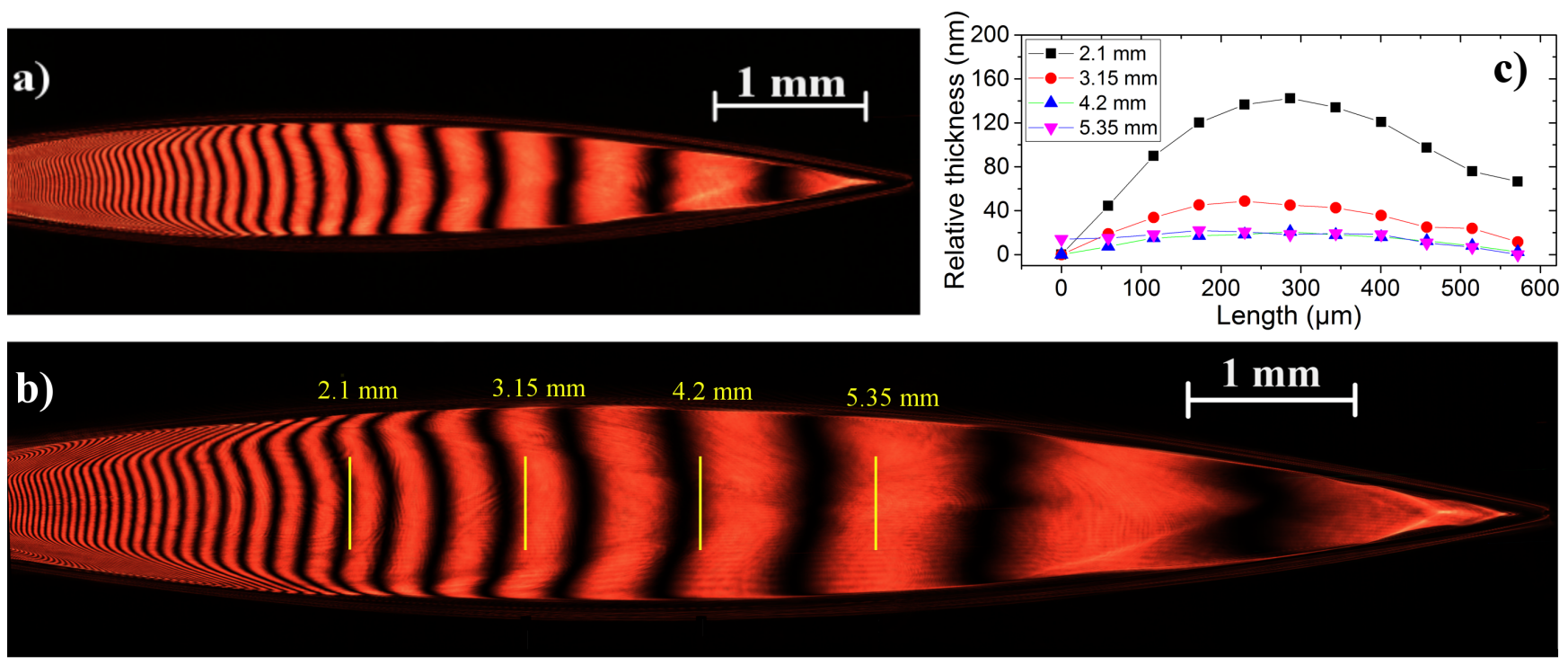

FIG. 6. A picture of the liquid isopropanol jet sheet for different flow rates: a) at the 2 bar stagnation pressure the flow rate was $7.4 \mathrm{~mL} / \mathrm{min}$ and $\mathbf{b}$ ) at the 3 bar stagnation pressure $-9.1 \mathrm{~mL} / \mathrm{min}$. In both cases an average of 5 images was taken. The images of the liquid sheet jets were recorded with a microscope objective $(10 \times$ magnification $)$ in sections and were combined (stitched) to form the full images. The nozzle is on the left and the jet flows from left to right. The jets were illuminated at a $15^{\circ}$ angle. This was the smallest angle at which the objective would not clip the incoming laser beam. In c) vertical sections of thickness profile are shown for a $9.1 \mathrm{~mL} / \mathrm{min}$ flow rate. The range and location of these measurements is marked as yellow lines in b). The profiles were acquired using the retrieved phase maps and show that at a distance of $2.1 \mathrm{~mm}$ the jet is $150 \mathrm{~nm}$ thicker in the center than at the edges of the measurement range. The flatness of the liquid jet surface improves with increasing distances from the nozzle.
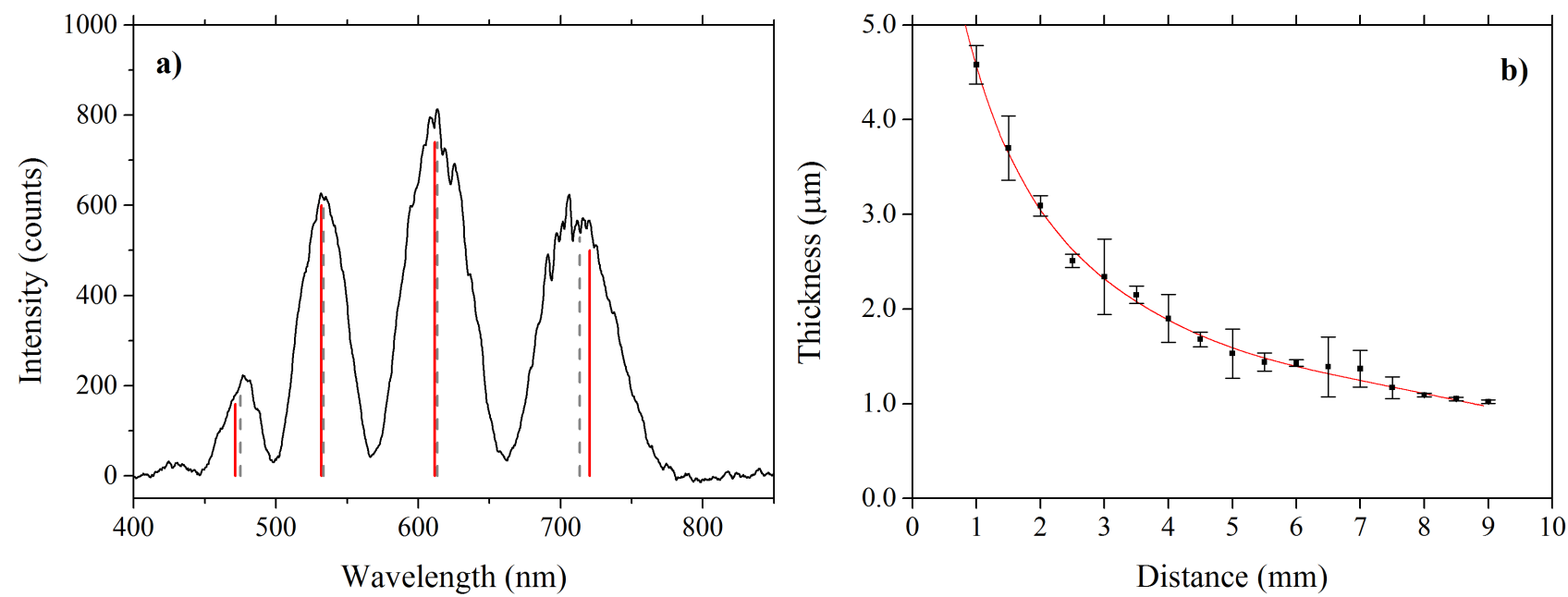

FIG. 7. An interferogram is shown in a) and thickness profiles for white light interferometry (scatter data points with error bars) and monochromatic interferometry (acquired from the retrieved phase map and shown as a continuous red line, below $1 \mathrm{~mm}$ distance the image resolution was insufficient to retrieve the phase map correctly) are shown and compared in $\mathbf{b}$ ). The continuous red line in b) was shifted vertically until it overlapped with the data points measured for white light interferometry. White light and monochromatic data were recorded on different days so slight variations in jet flow rate (flow rate defined by a pressure set by a pressure regulator) and length are expected. The data was recorded at about 3 bar backing pressure and $9.1 \mathrm{~mL} / \mathrm{min}$ flow rate (measured accurately for monochromatic interferometry). The interferogram was recorded at a distance of $4.5 \pm 0.05 \mathrm{~mm}$ from the nozzle. The baseline background subtraction and Savitzky-Golay smoothing with a 25-point-wide filter (the full width of the displayed spectrum is 1767 points) was performed. The exposure time was 200 ms. The peak locations in a) determined from the centre-of-mass analysis are shown as vertical dashed lines. These lines are marginally offset from the predicted positions of peaks for which each pair of peaks would give identical thickness (marked as a red continuous vertical line). At the $4.5 \pm 0.05 \mathrm{~mm}$ position the jet thickness was $1.68 \pm 0.08 \mu \mathrm{m}$. The thickness gradually decreased from $4.58 \pm 0.20 \mu \mathrm{m}$ to $1.02 \pm 0.02 \mu \mathrm{m}$ across the full length of the jet. 
sheet jet was rather uneven. We note that the Reynolds number for the water jet under similar conditions exceeds the threshold value of 2000 and therefore a transition to turbulent flow might be expected. For lower flow rates laminar flow can be regained, though the jet length is significantly decreased to $2-3 \mathrm{~mm}$. Further work is required to characterise water jets for this particular nozzle design. An optimisation of the geometry may be necessary to adapt the design of the nozzle to the lower viscosity fluids.

\section{Thickness characterization in atmosphere and in vacuum}

The peak values of the white light spectral interferograms were used in the analysis to determine the absolute thickness of the jet under normal pressure and in vacuum. The centre line and the centre point of the jet are most likely to be used for transient absorption measurements where the jet is flattest. Thickness measurements were undertaken for a flow rate of $3 \mathrm{bar}, 9.1 \mathrm{~mL} / \mathrm{min}$. The white light interference spectrum at about the centre point of the jet is shown in Fig. 7 a). The measurement was performed at a distance of $4.5 \pm 0.05 \mathrm{~mm}$ from the nozzle end and the local thickness was $1.68 \pm 0.08 \mu \mathrm{m}$. The thickness along the centre line for the same flow rate is shown in Fig. $\mathbf{7}$ b). A gradual decrease in thickness with the distance was observed. The same behaviour has been observed as for the colliding-jet-generated liquid sheets ${ }^{16}$ as well as other flat sheet experiments ${ }^{14,15}$.

The thickness measurement was repeated in vacuum for a $9.4 \mathrm{~mL} / \mathrm{min}$ flow rate. Due to the physical constraints of the set-up the full thickness profile along the centre line of the jet in vacuum could not be recorded without significant modifications to the experimental chamber. A measurement of the jet thickness in vacuum was undertaken at $4.97 \pm 0.02 \mathrm{~mm}$ distance from the nozzle at a pressure of $5 \times 10^{-1}$ mbar and the thickness of the jet was found to be $1.49 \pm 0.04 \mu \mathrm{m}$. This value matches within the error bars the measurement of thickness of $1.53 \pm 0.26 \mu \mathrm{m}$ at the corresponding distance in normal atmosphere. The observation is qualitatively similar to that observed by Ekimova et. al. ${ }^{16}, i$. e. the jet thickness does not differ significantly between vacuum and atmosphere.

\section{DISCUSSION}

The thin liquid sheets we have developed offer a number of technological benefits that enable a broad range of applications. The micrometer-thickness liquid jets we can create in vacuum can be used to improve temporal resolution of transient absorption measurements ${ }^{9-11}$ due to reduced path length through the target medium and thus reduced temporal walk-off between the pump and probe laser pulses. The liquid sheet targets can also reduce background absorption and could allow one to achieve better signal-to-noise ratio signals, in particular in the soft X-ray energy range where high absorption cross-sections are present. Likewise, the technology could enable HHG generation in liquids. The first attempts to explore this application were undertaken with rather thick $150 \mu \mathrm{m}$ water jets - and in consequence high re-absorption of the higher harmonics was a significant liming factor ${ }^{25}$. On the other hand two laser pulses can be used: the first pulse was used to ionise and explode microdroplets and the second laser pulse generated high harmonics with the delayed second laser pulse interacting with a high density vapour phase ${ }^{26}$. Few micrometer scale liquid targets can potentially overcome the reabsorption limitation. The thin liquid sheet target technology may also be applicable to fast ion acceleration ${ }^{18}$ or other experiments where laser damage is unavoidable - a flowing liquid target may be more practical than having to exchange a foil target every shot. The rapid and continuous formation of a new surface may also be attractive for deuteron acceleration from a liquid target for applications such as fusion neutron generation. In contrast, foil or tape systems require baking or laser cleaning to remove hydrocarbon contamination, which otherwise results in protons dominating the accelerated ion population, irrespective of the foil composition.

In future it would be valuable to carry out full fluid dynamics simulations to obtain further insights to explain the presence of standing waves or suggest how to further improve the nozzle design and thereby to enable use of fluids with a wider range of viscosities and surface tensions, including water, which is particularly attractive due to its great relevance in chemistry and biochemistry. First attempts with water resulted in rather uneven jets surfaces and steps can be made to improve the performance of these jets, for examples by reducing the Reynolds number such that a laminar flow is established. This can be achieved in two ways one of which is to reduce the jet velocity for this particular nozzle and then to undertake a full characterisation of thickness, optical flatness and other flow parameters. Another approach is to tailor the nozzle design and to change the slit length so that a more laminar flow regime is obtained. In practice this also means that the other dimensions and the nozzle shape may need modifications.

Many applications require liquid jets operating in vacuum at a level better than $5 \times 10^{-1}$ mbar. In our current experiment the pressure was limited by the effective pumping speed at the entrance port of the smaller 6-way cross chamber (see Fig. 2). In the future, the liquid jet will be placed in a custom liquid nitrogen trap enclosure within the vacuum chamber. The enclosure will have input and output ports for the laser beam or diagnostics and will be differentially pumped by the liquid nitrogen trap. In this way the laser beam will propagate through less vapour before it reaches the target. Also the working fluid can be pre-cooled or a solute added to reduce the vapour pressure.

Another important aspect to consider is condensation 
and flow of low-freezing-point solvents on the liquid nitrogen trap walls. Unlike water vapour, alcohols do not freeze-out so easily. The condensate was found to slowly flow down the surface of the liquid nitrogen trap then drip onto the vacuum chamber walls after which it vaporized causing an increase in base pressure. This was typically observed after 20 min operation of the liquid jet under vacuum conditions. A system for collecting condensate will be implemented in the future to eliminate this problem.

\section{CONCLUSIONS}

A novel method for producing 3D printed nozzles optimised for creating thin liquid jets was established and tested. The nozzles produced were stress-tested and did not display any structural damage at backing pressures of up to 8 bar. The flow of isopropanol through the nozzles was investigated under normal atmosphere and in vacuum and was found to create optically flat $<\lambda / 20$ surfaces over a length scale of $100 \mu \mathrm{m}$. For a flow rate of $9.1 \mathrm{~mL} / \mathrm{min}$ the thickness of the jet was in the range of 1.02-4.58 $\mathrm{\mu m}$ and the jet exhibited small-scale standing waves - fluid dynamics simulations will be required to fully explain this phenomena. Stable flow of the jet was likewise established in vacuum at a base pressure of $5 \times 10^{-1}$ mbar. The thickness of the jet in vacuum did not differ to that measured under normal atmosphere the jet thickness in vacuum was $1.49 \pm 0.04 \mu \mathrm{m}$. In contrast to the colliding circular jet method ${ }^{16}$ the set-up with a single 3D-printed nozzle is simple to align and maintain over long periods of time. The nozzles can be replaced and cleaned with relative ease. The creation of stable thin liquid jets in vacuum can be potentially used for a range of applications including transient absorption experiments in the soft X-ray energy range where micrometer-thick targets in vacuum are required due to high material absorption. The application of the jet is not limited to the transient absorption alone. The technology could potentially offer replenishing or self-healing targets for shock physics experiments or laser field accelerated proton or deuteron generation. Moreover, it may be used as a timing tool for XFEL experiments to complement SiN technology or potentially for high harmonic generation in liquids.

\section{ACKNOWLEDGEMENTS}

We are pleased to acknowledge the support of awards including EPSRC EP/I032517/1, ERC ASTEX 290467 and EPSRC MURI-MIR EP/N018680/1. A.B. acknowledges support from the Imperial College London Junior research fellowship.

${ }^{1}$ M. Faubel, B. Steiner, and J. P. Toennies, J. Chem. Phys. 106, 9013 (1997).

${ }^{2}$ M. Faubel, K. Siefermann, Y. Liu, and B. Abel, Accounts Chem. Res. 45, 120 (2011).

${ }^{3}$ A. T. Shreve, T. A. Yen, and D. M. Neumark, Chem. Phys. Lett. 493, 216 (2010).

${ }^{4}$ T. Gladytz, B. Abel, and K. R. Siefermann, Phys. Chem. Chem. Phys. 17, 4926 (2015).

${ }^{5}$ K. R. Wilson, B. S. Rude, T. Catalano, R. D. Schaller, J. G. Tobin, D. T. Co, and R. Saykally, J. Phys. Chem. B 105, 3346 (2001).

${ }^{6}$ J.-J. Velasco-Velez, C. H. Wu, T. A. Pascal, L. F. Wan, J. Guo, D. Prendergast, and M. Salmeron, Science 346, 831 (2014).

${ }^{7}$ J. Meibohm, S. Schreck, and P. Wernet, Rev. Sci. Instrum. 85, 103102 (2014).

${ }^{8}$ K. Kunnus, I. Rajkovic, S. Schreck, W. Quevedo, S. Eckert, M. Beye, E. Suljoti, C. Weniger, C. Kalus, S. Grübel, et al., Rev. Sci. Instrum. 83, 123109 (2012).

${ }^{9}$ C. Bressler, C. Milne, V.-T. Pham, A. ElNahhas, R. van der Veen, W. Gawelda, S. Johnson, P. Beaud, D. Grolimund, M. Kaiser, C. Borca, G. Ingold, R. Abela, and M. Chergui, Science 323, 489 (2009).

${ }^{10}$ N. Huse, H. Cho, K. Hong, L. Jamula, F. M. de Groot, T. K. Kim, J. K. McCusker, and R. W. Schoenlein, J. Phys. Chem. Lett. 2, 880 (2011).

${ }^{11}$ H. Cho, M. L. Strader, K. Hong, L. Jamula, E. M. Gullikson, T. K. Kim, F. M. de Groot, J. K. McCusker, R. W. Schoenlein, and N. Huse, Faraday Discuss. 157, 463 (2012).

${ }^{12}$ A. Johnson, L. Miseikis, D. Wood, D. Austin, C. Brahms, S. Jarosch, C. Strüber, P. Ye, and J. Marangos, Struct. Dyn. 3, 062603 (2016).

${ }^{13}$ M. Faubel, S. Schlemmer, and J. Toennies, Z. Phys. D Atom. Mol. Cl. 10, 269 (1988).

${ }^{14}$ A. Watanabe, H. Saito, Y. Ishida, M. Nakamoto, and T. Yajima, Opt. Commun. 71, 301 (1989).

${ }^{15}$ M. Kondoh and M. Tsubouchi, Opt. Express 22, 14135 (2014).

${ }^{16}$ M. Ekimova, W. Quevedo, M. Faubel, P. Wernet, and E. T. Nibbering, Struct. Dyn. 2, 054301 (2015).

${ }^{17}$ D. Hasson and R. E. Peck, Aiche J. 10, 752 (1964).

${ }^{18}$ R. Prasad, A. Andreev, S. Ter-Avetisyan, D. Doria, K. Quinn, L. Romagnani, C. Brenner, D. Carroll, N. Dover, D. Neely, et al., Appl. Phys. Lett. 99, 121504 (2011).

${ }^{19}$ D. A. Ventura and J. G. Nikelly, Anal. Chem. 50, 1017 (1978).

${ }^{20}$ A. Charvat, E. Lugovoj, M. Faubel, and B. Abel, Rev. Sci. Instrum. 75, 1209 (2004), http://dx.doi.org/10.1063/1.1710704.

${ }^{21}$ J. M. Cole, Diagnosis and application of laser wakefield accelerators, Ph.D. thesis, Imperial College London (2015).

${ }^{22}$ R. M. Goldstein, H. A. Zebker, and C. L. Werner, Radio Sci. 23, 713 (1988).

${ }^{23}$ S. Shin, J. Chergui, and D. Juric, arXiv preprint arXiv:1410.8568 (2014).

${ }^{24}$ S. Shin, J. Chergui, D. Juric, L. Kahouadji, O. K. Matar, and R. V. Craster, arXiv preprint arXiv:1702.02478 (2017).

${ }^{25}$ H. G. Kurz, D. S. Steingrube, D. Ristau, M. Lein, U. Morgner, and M. Kovačev, Phys. Rev. A 87, 063811 (2013).

${ }^{26}$ A. D. DiChiara, E. Sistrunk, T. A. Miller, P. Agostini, and L. F. DiMauro, Opt. Express 17, 20959 (2009). 